Further, the published research employs various injury definitions, data collection procedures and results presentation, limiting intergroup comparisons.

Objective To present health surveillance data from the Great Britain STSS (GBSTSS) elite training group across two competitive seasons and to recommend data collection and standardised injury and illness definitions.

Design Retrospective health surveillance report.

Setting Analysis of English Institute of Sport Performance Data Management System (PDMS) medical records alongside athlete reported training time and activity from GBSTSS athletes based in Nottingham, UK.

Patients (or Participants) Fifteen athletes from the GBSTSS World Class Programme squad. Nine males aged 17-33 years (mean $22.7 \pm 5.21$ ) and six females aged 17-26 (mean 22.7 $\pm 3.01)$.

Main Outcome Measurements Number of injuries/illness by body area, cause and incidence (per 1000 hrs of STSS).

Results In 2016-18 182 new injuries/illness were reported to the medical staff at GBSTSS. 126 of these (69\%) incurred any time loss/time restriction from training or competition. The sum of time loss/time restricted days was 1346 (illness and injury). The biggest threats to athlete availability in the GBSTSS programme were thigh, knee and lumbar spine injuries. The overall incidence per $1000 \mathrm{~h}$ of varied short track training and competition was 9.9 time loss episodes/1000 h (injury 4.4/1000 \& illness 5.5/1000).

Conclusions Injury had a larger impact on athlete availability in the GBSTSS despite the incidence of illness being greater. This data can be used to inform mitigation strategies in practitioners working to reduce time loss in the sport. STSS should follow other sports in agreeing definitions, methodologies and reporting procedures through consensus to improve consistency of data and intergroup comparisons, this should include incidence exposure based on hours of STSS activity.

\section{THE DESIGN AND IMPLEMENTATION OF SPORT INJURY SURVEILLANCE SYSTEM}

Mojtaba Ebrahimi Varkiani, Mohammad Hossien Alizadeh, Reza Rajabi, Hooman Minoonejad. University of Tehran, Tehran, Iran (Islamic Republic of)

\subsection{6/bjsports-2021-IOC.321}

Background The increase in sport injuries is a big challenge in public health which requires preventive measures. To reach a successful prevention, implementation of an injury surveillance system is the first and important step.

Objective The present study aimed to design and implement a sport injury surveillance system.

Design Athletic trainers recorded athlete`s sport injuries in soccer, volleyball, handball, taekwondo and wrestling in a period of 6 months via a comprehensive sport injury surveillance system prospectively, using a web-based sport injury recording application on smart phones designed for athletic trainers to collect data.

Setting The pilot study was implemented in Alborz province of Iran. Finally, results were compared with the previous sport injury recording system.

Patients (or Participants) All professional and nonprofessional men and woman included.

Main Outcome Measurements Collecting injuries via surveillance
Results 81 sport injuries were reported in surveillance system. An incidence rate of 1.39 injuries per 1000 athletes was registered in the surveillance system, while there was 0.32 injury per 1000 athletes registered in the sport injury recording system with 19 reported injuries. Contusions and bruising were the most common types of injuries. Finger and knee injuries were the most common body parts in sport injury surveillance system, while knee and thigh injuries were most common in sport injury recording system. There was a significant difference between the results of two systems as well $(\mathrm{p}<0.05)$.

Conclusions Sport injury recording was done using a comprehensive web-based system for the first time in Iran. Different implementation and reporting methods and having injury definition may affect the results. Use of injury record application on cellphones may facilitate injury recording as well. Use of surveillance system with successful implementation is a rerequisite for effective prevention, especially in a sports population. Application of simple and user friendly tools and providing acceptance and developing prevention culture in athletes and coaches are necessary to succeed in injury surveillance.

\section{THE SWISS REGISTRY OF FATALITIES IN SPORTS: HOW TO OVERCOME DATA GAPS}

Steffen Niemann, Philip Derrer, Flavia Buergi, Mirjam Baechli, Othmar Bruegger. Swiss Council for Accident Prevention BFU, Bern, Switzerland

\subsection{6/bjsports-2021-IOC.322}

Background Fatality tops the injury pyramid. Knowledge of the extent and nature of fatal sports injuries is a key element in prevention. However, those data were not available for a long time in Switzerland. Like other countries, Switzerland has a mortality database, but with 3 major shortcomings: it is almost impossible to identify sports fatalities with the International Statistical Classification of Diseases and Related Health Problems 10th version (ICD-10) external causes; data are entered with a time lag of three years; and fatal injuries in tourists are not recorded.

Objective To estimate the extent of sports fatalities in Switzerland.

Design We retrieved data from a registry of sports fatalities established in 2000, by the Swiss Council for Accident Prevention BFU. The main source of data was a standardized query, conducted by a Swiss media agency. The registry was supplemented and validated with data from partner organizations, like the Swiss Alpine Club or the Swiss Life Saving Organization. Sports, age, sex, date, time, location of fatal injury, and numerous other characteristics were encoded.

Setting Recreational sports.

Results Since 2000, 3397 fatalities were registered (179 annually, SD: $26 ; 83 \%$ males). The mean age was 45 y (SD: 19). Among the fatally injured, $32 \%$ were tourists from abroad. Most deaths occurred while mountain hiking, mountaineering, and climbing, followed by swimming in open water and backcountry skiing.

Conclusions The sports fatality registry established by the Swiss Council for Accident Prevention is an important, valid supplement to existing injury data. It allows detailed, up-todate analyses of fatal sports injuries, which supports the priorities of the BFU's nationwide prevention strategy. 